\title{
The global epidemiology of chronic cough in adults: a systematic review and meta-analysis
}

\author{
To the Editor:
}

Cough is an essential defence mechanism [1]. However, chronic cough is a significant cause of morbidity, seriously impairing quality of life [2]. Previously, chronic cough was considered a consequence of various diseases, such as asthma/eosinophilic bronchitis, rhinitis and gastro-oesophageal acid reflux disease $[3,4]$. Recent evidence, however, suggests that chronic cough is a clinical syndrome with distinct intrinsic pathophysiology characterised by neuronal hypersensitivity [5-7]. Here, we estimated the worldwide epidemiological burden of chronic cough irrespective of putative diagnosis in general adult populations using a comprehensive systematic literature review.

We searched the literature for prevalence of chronic cough in community-based adult populations using Pubmed and Embase databases according to the recommendations of the PRISMA statement [8]. The search terms were "cough AND (epidemiology OR epidemiologic OR epidemiological OR prevalence OR incidence)" for articles published in peer-reviewed journals between 1980 and 2013. An additional search was performed in Google Scholar and via cross-referenced articles. Language was not restricted. If full-text links were not available, we contacted the corresponding authors by e-mail.

Inclusion criteria were as follows: 1) cross-sectional or longitudinal studies conducted in community-based or unselected adult populations, and 2) reported prevalence of chronic cough. Owing to the lack of a validated epidemiological classification for chronic cough [9], various definitions were accepted. Exclusion criteria were as follows: 1) no relevant analyses, 2) convenience sample studies without detailed information, 3) birth cohort studies, 3) specific occupation studies, 4) studies using duplicate samples, or 5) non-original papers. In determining eligibility, discrepancies were resolved by consensus among the authors. For all the included articles, data were extracted by researchers, and confirmed by W-J. Song and Y-S. Chang. The extracted outcomes included research question, study design, study region, study year, participant characteristics and case definition.

We adapted a quality effects model for meta-analyses. Heterogeneity is the main issue with meta-analysis of prevalence, probably due to a combination of true variance in the prevalence and variability produced by the methodological differences used to measure the outcome [10]. The quality effects model has strengths in that it gives more weight to studies of high quality, as it uses quality scores assigned to each study [10]. Briefly, the quality of the included studies were calculated by summation of six items: 1) definition of the population, 2) use of reported diagnostic criteria, 3) method of case ascertainment, 4) administration of measurement protocol, 5) characteristics of catchment area, and 6) prevalence measure, according to the developer's protocol [10].

For meta-analyses, the numbers of subjects with chronic cough in each study were combined. Homogeneity testing was performed using the $\mathrm{I}^{2}$ test. Correlation between chronic cough and smoking prevalence was evaluated by Spearman's test. Relationships of chronic cough with region and smoking were examined using meta-regression. Quality effects model analyses were performed using MetaXL 2.2 (http://www.epigear.com); and all other statistical analyses were conducted by using Stata 12.0 (Stata Corp., College Station, TX, USA).

A total of 17891 citations were initially identified by the search strategy, and 398 full-text articles were retrieved after screening. 90 studies were finally selected. In pooled analyses, the overall prevalence of chronic cough was 9.6\% (95\% CI 7.6-11.7\%; I²=99\%; 576839 subjects). The regional prevalences were higher in Oceania (18.1\% (95\% CI 9.8-27.2\%) in 5541 subjects from three studies), Europe (12.7\% (95\% CI 10.4-15.2\%) in 344522 subjects from 44 studies) and America (11.0\% (95\% CI 7.8-14.4\%) in 60585 subjects from 14 studies) than in Asia (4.4\% (95\% CI 1.8-7.4\%) in 131566 subjects from 22 studies) and Africa (2.3\% (95\% CI $0.0-6.7 \%)$ in 34625 subjects from seven studies). The pooled prevalence according to country is presented in figure 1 . 


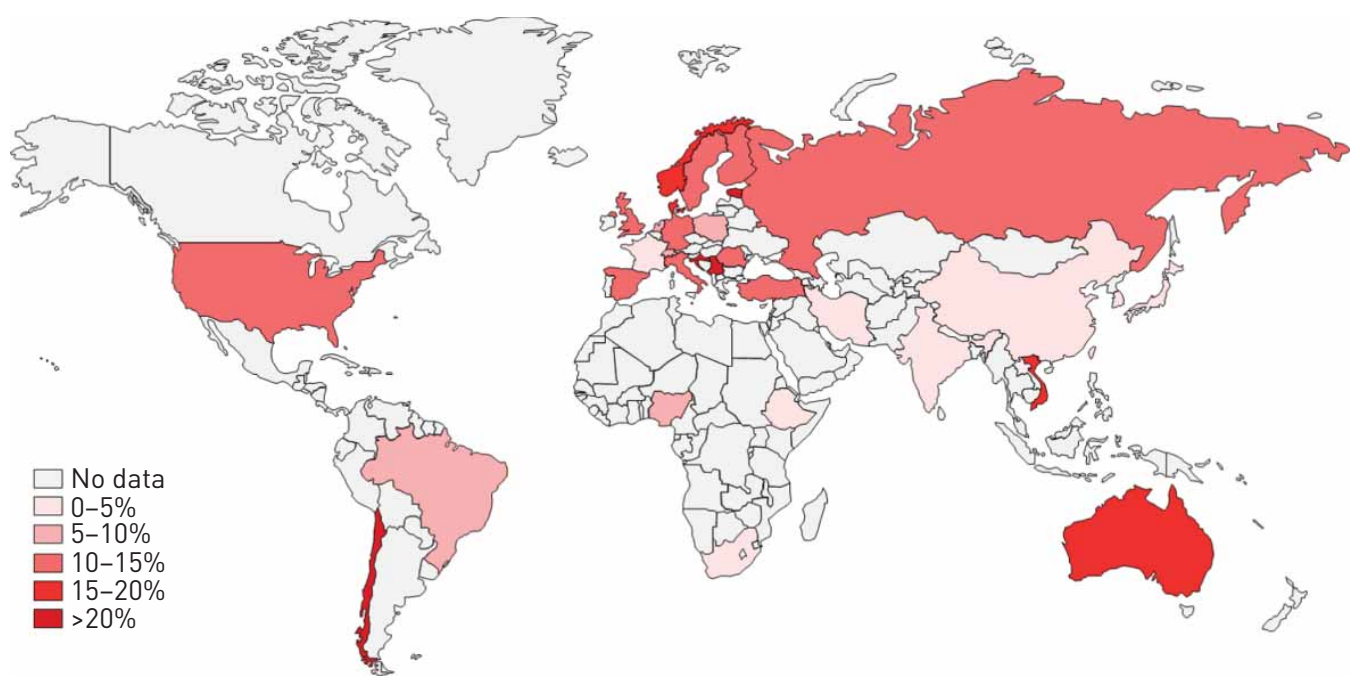

FIGURE 1 Map showing the pooled prevalence of chronic cough by country.

The prevalence of current smoking correlated with cough prevalence $(\mathrm{r}=0.501, \mathrm{p}<0.001)$. However, in the meta-regression analyses, the regional differences in chronic cough prevalence were independent of smoking.

Only 16 studies analysed chronic cough as the primary outcome, while the remaining majority included chronic cough as part of a general inquiry into respiratory symptoms. A total of 19 different definitions were utilised among 90 studies. The most common definitions were "cough $\geqslant 3$ months (such as "Do you usually cough on most days for 3 consecutive months or more during the year?")" (n=42) and "cough $\geqslant 3$ months for 2 successive years" ( $n=16)$; they had similar pooled prevalences of $7.9 \%$ (95\% CI 4.8 $\left.11.2 \% ; \mathrm{I}^{2}=100 \%\right)$ and $7.9 \%$ (95\% CI $\left.5.2-10.9 \% ; \mathrm{I}^{2}=99 \%\right)$, respectively. Only three studies utilised the 8-week cut-off duration [11-13], reporting the prevalence as $12.0 \%$ (UK), 2.2\% (Japan), and $1.1 \%$ (Nigeria), respectively. Considering the heterogeneity in the definitions, we repeated the meta-regression analyses in a subsample of these 58 studies using two common definitions, and found the relationships between region and chronic cough prevalence to be consistent.

The present study is the first systematic review and meta-analysis to estimate the epidemiological burden of chronic cough in general adult populations. The primary finding was a high global prevalence (9.6\%), comparable to that reported for asthma or chronic obstructive pulmonary disease $[14,15]$. Heterogeneity in the definitions may have biased the prevalence. However, chronic cough showed a $7.9 \%$ prevalence, even when the most stringent temporal definition, "cough $\geqslant 3$ months" was used. Therefore, chronic cough appears to be a global, but poorly recognised, condition causing significant morbidity.

Chronic cough was significantly more frequent in Europe and America than in Asia and Africa. DicPINIGAITIS et al. [16] found no ethnic difference in cough reflex sensitivity between Caucasian, Indian or Chinese subjects using capsaicin cough challenge. Therefore, ethnic or genetic differences in cough reflex sensitivity are unlikely to be solely responsible for this regional variation. Environmental factors may account for the regional variation; urbanisation in Western countries may increase the risk of inhalational exposure to irritants. Supporting this hypothesis, we found significant correlations between smoking and chronic cough prevalence. Comorbidities such as obesity or rhinitis might also contribute to the regional variability [11, 17]; however, we were unable to examine these relationships, since relevant outcomes were reported in relatively few studies.

Future investigation into the epidemiology of chronic cough will require the development of an international consensus definition. As shown here, more than $80 \%$ of previous studies were not primarily intended to study chronic cough, and 19 different definitions were utilised. Current clinical guidelines define chronic cough using an arbitrary cut-off duration of 8 weeks $[3,4]$. However, the majority of the epidemiological studies in the existing literature used a 3-month cut-off. Prospective investigations into the temporal characteristics of acute and chronic cough will be required to inform evidence-based definitions of the clinical syndromes.

Several limitations need to be considered. First, the definitions were heterogeneous, and thus the prevalence estimation might have been biased. The majority of included studies utilised the 3-month cut-off duration, and thus the estimated prevalence may not be directly applied to chronic cough in clinical guidelines (cough $\geqslant 8$ weeks). However, the prevalence data from three studies using the 8 -week cut-off duration were in line with their corresponding regional prevalence in general. Secondly, relatively few of the studies were conducted in non-European countries, which might have skewed the global 
prevalence estimation, particularly because of their lack of standardised definitions. To compensate for this heterogeneity, we performed sub-analyses by case definitions, and found similar degrees of prevalence and consistent patterns of regional association.

Despite these limitations, the present study is the first systematic analysis to estimate the epidemiological burden of chronic cough. We included all the available literature published during the last three decades, with no language restriction in study selection. Such inclusivity allowed the examination of regional difference. We identified the methodological inconsistencies in the existing literature and suggest that there is an urgent need for global standardisation.

In conclusion, the high epidemiological burden of chronic cough demonstrated here represents a major unmet clinical need.

(?) @ERSpublications

Chronic cough has a high global epidemiological burden. A standard definition would facilitate further studies. http://ow.ly/HPCnw

Woo-Jung Song ${ }^{1}$, Yoon-Seok Chang ${ }^{1,2}$, Shoaib Faruqi ${ }^{3}$, Ju-Young Kim ${ }^{1}$, Min-Gyu Kang ${ }^{1}$, Sujeong Kim ${ }^{4}$, Eun-Jung Jo ${ }^{5}$, Min-Hye Kim ${ }^{6}$, Jana Plevkova ${ }^{7}$, Heung-Woo Park ${ }^{1}$, Sang-Heon Cho ${ }^{1,8}$ and Alyn H. Morice

${ }^{1}$ Dept of Internal Medicine, Seoul National University College of Medicine, Seoul, Korea. ${ }^{2}$ Dept of Internal Medicine, Seoul National University Bundang Hospital, Seongnam, Korea. ${ }^{3}$ Centre for Cardiovascular and Metabolic Research, Hull York Medical School, Castle Hill Hospital, University of Hull, Cottingham, UK. ${ }^{4}$ Dept of Internal Medicine, Kyungpook National University School of Medicine, Daegu, Korea. ${ }^{5}$ Dept of Internal Medicine, Pusan National University College of Medicine, Busan, Korea. ${ }^{6}$ Dept of Internal Medicine, Ewha Woman's University School of Medicine, Seoul, Korea. ${ }^{7}$ Dept of Pathophysiology and Simulation Centre, Jessenius Faculty of Medicine, Comenius University, Bratislava, Slovak Republic. ${ }^{8}$ Both authors contributed equally; S-H. Cho and A.H. Morice are joint lead authors of this article.

Correspondence: Alyn H. Morice, Cardiovascular and Respiratory Studies, University of Hull, Hull York Medical School, Castle Hill Hospital, Cottingham, HU16 5JQ, UK. E-mail: a.h.morice@hull.ac.uk

Received: Sept 232014 | Accepted after revision: Dec 242014 | First published online: Feb 052015

Conflict of interest: None declared.

Acknowledgements: We gratefully acknowledge the help of GlaxoSmithKline (UK) in providing data translation assistance. We also wish to acknowledge the statistical advice from Soyeon Ahn (Division of Statistics, Medical Research Collaborating Center, Seoul National University Bundang Hospital, Seongnam, Korea).

\section{References}

1 Brooks SM. Perspective on the human cough reflex. Cough 2011; 7: 10.

2 Birring SS, Prudon B, Carr AJ, et al. Development of a symptom specific health status measure for patients with chronic cough: Leicester Cough Questionnaire (LCQ). Thorax 2003; 58: 339-343.

3 Morice AH, Fontana GA, Sovijarvi AR, et al. The diagnosis and management of chronic cough. Eur Respir J 2004; 24: 481-492.

4 Irwin RS, Baumann MH, Bolser DC, et al. Diagnosis and management of cough executive summary: ACCP evidence-based clinical practice guidelines. Chest 2006; 129: 1S-23S.

5 Chung KF, McGarvey L, Mazzone SB. Chronic cough as a neuropathic disorder. Lancet Respir Med 2013; 1: 414-422.

6 Morice AH, Millqvist E, Belvisi MG, et al. Expert opinion on the cough hypersensitivity syndrome in respiratory medicine. Eur Respir J 2014; 44: 1132-1148.

7 Morice $\mathrm{AH}$, Jakes AD, Faruqi S, et al. A worldwide survey of chronic cough: a manifestation of enhanced somatosensory response. Eur Respir J 2014; 44: 1149-1155.

8 Moher D, Liberati A, Tetzlaff J, et al. Preferred reporting items for systematic reviews and meta-analyses: the PRISMA statement. Ann Intern Med 2009; 151: 264-269.

9 Liard R, Neukirch F. Questionnaires: a major instrument for respiratory epidemiology. ERS Monogr 2000; 15: 154-166.

10 Barendregt JJ, Doi SA, Lee YY, et al. Meta-analysis of prevalence. J Epidemiol Community Health 2013; 67: 974-978.

11 Ford AC, Forman D, Moayyedi P, et al. Cough in the community: a cross sectional survey and the relationship to gastrointestinal symptoms. Thorax 2006; 61: 975-979.

12 Desalu O, Salami A, Fawibe A. Prevalence of cough among adults in an urban community in Nigeria. West Afr J Med 2011; 30: 337-341.

13 Fujimura M. Frequency of persistent cough and trends in seeking medical care and treatment-results of an internet survey. Allergol Int 2012; 61: 573-581.

14 Anandan C, Nurmatov U, Van Schayck O, et al. Is the prevalence of asthma declining? Systematic review of epidemiological studies. Allergy 2010; 65: 152-167.

15 Halbert RJ, Natoli JL, Gano A, et al. Global burden of COPD: systematic review and meta-analysis. Eur Respir J 2006; 28: 523-532.

16 Dicpinigaitis PV, Allusson VR, Baldanti A, et al. Ethnic and gender differences in cough reflex sensitivity. Respiration 2001; 68: 480-482.

17 Song WJ, Morice AH, Kim MH, et al. Cough in the elderly population: relationships with multiple comorbidity. PLoS One 2013; 8: e78081. 